\title{
Heilmittel noch ein Quartal mit alter Software verordnen
}

Seit dem 1. Januar 2017 darf für die Verordnung von Heilmitteln nur noch zertifizierte Software eingetzt werden. Im 1. Quartal 2017 kann übergangsweise aber noch eine nicht zertifizierte Software genutzt werden.

Die neue Verordnungssoftware enthält alle Informationen der Heilmittelrichtlinie inklusive des Heilmittelkatalogs auf dem aktuellen Stand. Sie prüft die Plausibilität der eingegebenen Daten und gibt Hinweise, wenn das Formular unvollständig oder die Verordnungsmenge zu hoch ist, oder wenn bei einer Verordnung außerhalb des Regelfalls die medizinische Begründung fehlt. Sie zeigt auch verbindlich an, wenn eine Praxisbesonderheit oder ein langfristi- ger Heilmittelbedarf begründet ist. Mit der Zertifizierung wurde die KBV beauftragt (siehe MMW 21-22/2016).

\section{MMW-KOMMENTAR}

Offensichtlich hat es mit dem geplanten Abschluss der Zertifizierung bis Ende des Jahres nicht geklappt, sodass diese Übergangsregelung vereinbart werden musste. Sie sieht vor, dass im 1. Quartal 2017 auch eine nicht zertifizierte Praxisverwaltungssoftware für die Verordnung von Heilmitteln verwendet werden kann. Ab dem 2. Quartal 2017 muss bei der Sammelerklärung zur Quartalsabrechnung dann bestätigt werden, dass in der Praxis eine zertifizierte Software zur Heilmittelverordnung verwendet wird. Ansonsten ist eine Verordnung nicht mehr möglich!

\section{Bei Lymphödemen bleibt Physiotherapie regressfrei}

KBV und Kassen haben vereinbart, dass bestimmte Lympherkrankungen mit langfristigem Heilmittelbedarf ab Januar 2017 übergangsweise als Diagnosen mit besonderem Verordnungsbedarf anerkannt werden. Dabei geht es um Lymphödeme im Stadium III an den Extremitäten (ICD-10-Code I89.02) oder einer sonstigen Lokalisation (I89.05) sowie hereditäre Lymphödeme (Q82.0-). Die dafür verordnete Physiotherapie LY2 geht somit nicht in die Verordnungsstatistik der Praxis ein.

\section{MMW-KOMMENTAR}

Im Zuge der jährlichen Revision der bundesweit geltenden Diagnoseklassifikation ICD-
10-GM für das Jahr 2017 wurden die Codes I89.0 für nicht klassifizierte Lymphödeme (Elephantiasis) und Q82.0 für hereditäre Lymphödeme aufgegliedert. Dadurch können hier nun auch der Schweregrad und die Lokalisation verschlüsselt werden. Diese Änderungen konnte vom Gemeinsamen Bundesausschuss (G-BA) allerdings nicht fristgerecht zum 1. Januar 2017 in die Diagnoseliste für den langfristigen Heilmittelbedarf aufgenommen werden.

Die vorübergehende Aufnahme in die Liste der besonderen Verordnungsbedarfe soll sicherstellen, dass Verordnungen aufgrund dieser Erkrankungen in der neuen Software verarbeitet werden können - und auch weiterhin keine Regresse verursachen. 